\title{
The Implications of a Pacing Guide on the Development of Students Ability to Prove in Geometry
}

\author{
Ruthmae Sears ${ }^{1 *}$
}

\author{
${ }^{1}$ University of South Florida, USA \\ *CORRESPONDENCE: $\square$ ruthmaesears@usf.edu
}

\begin{abstract}
This study examined the influence of a departmental decision to use the same pacing guide on the planning and enactment of proof tasks of the district-adopted textbook (Prentice Hall Geometry). Quantitative data were collected from a textbook analysis and the tasks students were assigned, and the qualitative data were collected from classroom observations, teachers' artifacts and interviews. The results indicate that teachers adhere to their departmental pacing guide by assigning the same tasks, however, there existed variation in the enacted lessons. Additionally, the results suggest the proof tasks assigned in the pacing guide generally required little cognitive rigor. This study has implication on the development of students' proof skills.
\end{abstract}

Keywords: geometry, proof, textbook, planning guide

\section{INTRODUCTION}

In geometry, the instructional responsibilities of geometry teachers are vast, and teaching proof is just one of them, hence the way that teachers use the textbook should seek to promote a holistic understanding of core concepts while promoting Standards of Mathematical Practices (CCSSM, 2010). There are many things in a geometry curriculum that have to be taught so teachers have to consider relative to proof: aspects of proof that need to be accentuated, potential pedagogical strategies that can bridge deductive thinking and geometric insight, and tools that can facilitate students' learning to prove (Jones, 2000). According to Jones (2002), "Teaching geometry well involves knowing how to recognize interesting geometrical problems and theorems, appreciating the history and cultural context of geometry, and understanding the many and varied uses to which geometry is put" (p.122).

Additionally, sociomathematical norms in a classroom can influence how proof is taught. There is a reflexive relationship between students' perceptions of their role, the teacher's role, classroom social norms and what is deemed mathematical activity (Yackel, 1996). "Doing proofs" embodies various actions by teachers and students which are influenced by stated or implicit norms of what work is valued, the structure in which proof ought to be presented, the time allocation for proving, and the responsibility of students and teachers while "doing proofs" (Herbst, 2009).

Notwithstanding that teaching can be socially influenced (Brown, 2011), in examining how proof is taught in geometry, thought must be given to the textbook as well as planning initiatives and curriculum goals for the school. Researchers have documented that teachers may use curriculum materials in different ways because of their conceptions about the curriculum, teaching, and teacher-curriculum relationships (Lloyd, 2009; Remillard, 2005; Remillard, 2009). If a minuscule focus is given to proof in the textbook or the lesson

\footnotetext{
Article History: Received 19 April $2018 \bullet$ Revised 31 July $2018 \bullet$ Accepted 3 August 2018

(C) 2018 The Author(s). Open Access terms of the Creative Commons Attribution 4.0 International License (http://creativecommons.org/licenses/by/4.0/) apply. The license permits unrestricted use, distribution, and reproduction in any medium, on the condition that users give exact credit to the original author(s) and the source, provide a link to the Creative Commons license, and indicate if they made any changes.
} 
planning, it is highly unlikely that the orchestrated discourse within the enacted lesson will promote students engagement with proof. Therefore, this study sought to answer the following research question:

- How does a geometry team pacing guide, which identifies tasks from the district adopted textbook for students to practice mathematical ideas, influence the planning and enactment of proof tasks?

\title{
LITERATURE REVIEW
}

Teachers' usage of curriculum materials embodies various pedagogical actions; teachers' can exhibit a reliance on curriculum materials for lesson planning, and the enacted lesson, or interact with curriculum materials as a form of resources (Lloyd, 2009). Remillard (2005) and Stein et al. (2007) have described teachers' use of curriculum materials as following or subverting, drawing on, interpretation, and participating with. The extent teachers' use their textbook may be influenced based on textbook features (tasks, structure, embedded teacher support and pedagogical emphasis) and teacher resources (Human capital, social capital and agency) (Remillard, 2009). Although teachers make the final decision as to what will be taught in the enacted lesson, they receive messages about what should be taught from different sources: curriculum materials, professional development, assessments, school hierarchy, parents, other teachers, administrators, district supervisors, and from their own experiences (Porter, 2002). Hence teachers' draw on curriculum materials for insight as to what to teach, but curriculum materials are not the only variable that contributes to what is taught.

Teaching proof embodies a "social character" (Alibert, 1991). In facilitating proof, teachers often provide a task with sufficient information, encourages students to provide appropriate reasoning, and provide opportunities for students to share ideas, as well as practice proving during instructional time (Herbst, 2009; Fussell, 2005). Within school mathematics:

\begin{abstract}
Proof is a mathematical argument, a connected sequence of assertions for or against a mathematical claim, with the following characteristics: 1. It uses statements accepted by the classroom community (set of accepted statements) that are true and available without further justification; 2. It employs forms of reasoning (modes of argumentation) that are valid and known to, or within the conceptual reach of, the classroom community; and 3. It is communicated with forms of expression (modes of argument representation) that are appropriate and known to, or within the conceptual reach of the classroom community \{Stylianides, 2008, p. 291).
\end{abstract}

The socio-mathematical norms within the classroom can influence students' opportunity to engage in reasoning and proof (Martin, 2005). For instance, Heinze and Reiss (2009) found that the 7th and 8th grade students' performance on the pretest and posttest was correlated more strongly at the classroom level $(\mathrm{r}=0.604$, $\mathrm{p}<0.001)$ than to the individual $(\mathrm{r}=0.435, \mathrm{p}<0.001)$. Nevertheless, the researchers concluded that students' proof and argumentation skills are weak across secondary grade levels.

Notwithstanding the classroom community can influence how textbooks are used and how proof is taught, salient factors such as teachers' belief, teachers' knowledge and the professional community can have impact on instruction as well. Philipp (2007) noted, "Teachers' affect is not nearly as important as teachers' beliefs" (p. 309). Some teachers believe that proof is a form of convincing or a way to promote understanding (Furinghetti, 2011). Knuth (2002) conducted semi-structured interviews with 16 in-service high school mathematics teachers about their conceptions of proof in mathematics. He found that $75 \%$ of teachers considered that the role of proof was to communicate mathematics, $50 \%$ of teachers considered that it was to systematize mathematical ideas and construct new knowledge, $18.75 \%$ considered proof as a means of explaining (answering why), and no teacher considered proof a means of explanation that can promote understanding. Additionally, Knuth (2002) found that $37.5 \%$ of teachers believed a proof became "invalid" if there was a contradictory statement, $31.25 \%$ of teachers were hesitant to accept a counterexample as a proof, and that $31.25 \%$ of teachers believed that unusual cases of counterexamples ought to be tested. Furthermore, Knuth (2002) showed that teachers identified characteristics of a convincing proof primarily in terms of concrete features (81.25\% of teachers), familiarity (62.5\%), generality (56.25\%) and amount of details (50\%). Knuth's results emphasized that teachers consider proof as a means to communicate mathematics, and that concrete features of proof are of utmost importance.

Many teachers may not possess the knowledge needed to construct proof. Schwarz and Keiser (2009) found that many preservice teachers were not able to construct proof that used lower-secondary mathematics content. Similarly, Brown and Stillman (2009) found that not all preservice teachers could recognize the 
generality of proofs. Teachers' fragile understanding of proof may cause them to avoid teaching it, or potentially limit the rigor of proof tasks teachers pose.

Additionally, community of practice can influence teachers' belief and practices (Philipp, 2007; Stein, 2007) relative to proof. The community can impact teachers' professional learning and adaptation of new teaching strategies (Cobb, 2003; Franke, 2001). Therefore, a teacher's identity within the school context is not static within the environment and is developed based on social interactions (Brown, 2011; Spillane, 2000).

\section{METHODS}

This study, which seek to examine how the departmental pacing guide influenced teachers' planning and enactment of proof tasks within the district adopted textbook, was conducted within the Midwest region of the United States. Data were collected via multiple sources. The quantitative data were collected from a textbook analysis and the tasks students were assigned, while the qualitative data were collected from teachers' interviews, artifacts and classroom observations.

Admittedly, teaching is a complex system, and proof is a mathematical process that is not always fully conceptualized (Healy, 2000; Knuth, 2009). Hence, two teachers employed at the same school, were used to provide insight as to how a pacing guide influenced their planning and enactment of proof tasks. Taking into account the uniqueness of the teachers' instructional practices and the variation that may exist among them (such as differences in experiences, mathematical knowledge pertinent to proof, and class structure), each teacher was analyzed individually and subsequently a cross case analysis was employed. Thus, this methods section will describe the textbook used, the collaborative initiative, the participants and how the data were collected and subsequently analyzed.

\section{Textbook}

The Midwestern school district adopted textbook was Prentice Hall Geometry (Bass et al, 2004). The textbook has chapters with multiple sections. Each of the sections has a lesson preview, which identifies the learning objectives, examples, and practice exercises, standardized test prep and mixed review tasks. In the back of the textbook additional resources are provided, such as: Skill handbook, geometric tables, postulates, theorems and constructions, and answers to selected geometrical problems.

Three chapters in the textbooks were analyzed for tasks features and levels of cognitive demands (Henningsen, 1997). The chapters examine were Chapter 2- Reasoning and Proof, Chapter 3- Parallel and perpendicular lines and Chapter 4 - Congruent Triangles. Chapter 2, entitled "Reasoning and proof", consists of five sections: Conditional statements, Biconditionals and Definitions, Deductive Reasoning, Reasoning in Algebra, and Proving angles congruent. Chapter 3, "Parallel and perpendicular lines", is comprised of seven sections: Properties of parallel lines, Proving lines parallel, Parallel lines and the triangle angle-sum theorem, The polygon angle-sum theorems, Lines in the coordinate plane, Slope of parallel and perpendicular lines, and Constructing parallel and perpendicular lines. Whereas, Chapter 4, "Congruent triangles", has seven sections: Congruent Figures, Triangles congruence by SSS and SAS, Triangle congruence by ASA and AAS, Using congruent triangles: CPCTC, Isosceles and equilateral triangles, Congruence in right triangles, Using corresponding parts of congruence triangle. The three chapters were selected because the teachers noted these were the chapters students were generally taught about proof and the research literature suggests such there exist a likelihood that students can be exposed to proof for these mathematical topics (Donoghue, 2003; Herbst, 2002).

\section{Departmental Pacing Guide}

At the school, the geometry team utilized a departmental pacing guide for over four years, which provided an outline of the sections within each chapter of the textbook, which ought to be addressed and the number of days that should be allocated for each section. Additionally, the pacing guide identified explicit tasks within the textbook that students should complete to practice mathematical concepts, and to prepare for end of chapter tests. The pacing guide noted whether a worksheet would be provided, and whether students will receive a test review packet. Teachers had the flexibility to alter lessons if needed. For Chapters 2-4, two sections were not assigned, namely: Section 2.3- Deductive Reasoning and Section 4.4- Using Congruent Triangles CPCTC. Generally, most topics were allocated one instructional day, however three sections were allocated two instructional days. The sections that obtained a greater allocation of time were: Section 3.2 - 
Proving lines parallel, Section 3. 4- The polygon angle sum theorem, and Section 3.6 - Slopes of parallel and perpendicular lines.

\section{Teachers}

The teachers (Mrs. Davis and Mrs. Bethel - pseudonyms) that participated in the study used the Prentice Hall Geometry (Bass, Charles, Johnson, \& Kennedy, 2004) textbook for more than three years, earned a graduate degree in curriculum and instruction, and were highly recommended by their mathematics department as model geometry teachers. The teachers worked at a large urban school (with a population greater than 1,800 students) and taught classes on a block schedule (88 minutes per lesson). Mrs. Davis has taught geometry for 6 years and since Prentice Hall Geometry (Bass, Charles, Johnson, \& Kennedy, 2004) is the sole curriculum that she ever used to teach geometry, she had limited knowledge of other curriculum to compare the district adopted textbook. Mrs. Bethel has 18 years' experience teaching mathematics, 15 years of which were devoted to teaching geometry. Unlike Mrs. Davis, Mrs. Bethel has utilized multiple curriculum materials over the years. I observed Mrs. Davis for 6 lessons and Mrs. Bethel for 8 lessons respectively.

\section{Interviews}

Teachers were interviewed prior to classroom observations to obtain information about their academic and professional background, proof conceptions, the need for proof in school mathematics and the extent proof related activities were emphasized in their instructional practices. Additionally, teachers were interviewed informally throughout the semester about instructional decisions made or about a particular feedback that was provided to students during the enacted lessons. Most of the interviews were audio recorded. The few instances the interviews were not recorded were during break periods, where the teachers were transient between their classrooms and the teachers' lounge.

\section{Classroom Observation Protocol}

Using a case study provided a holistic and context sensitive means to examine how geometry teachers use their district-adopted textbook and the pacing guide to teach proof. I observed the geometry lessons as a whole as well as focused my attention to the proof tasks used during instruction. For example, in a lesson a teacher may allocate a small percentage of class time to proof, and use a greater amount of time to proof-related or other geometry activities. Hence, I paid careful attention to the possibility that the course discussions can promote students constructing proof, even if an explicit proof task was not posed. An observational protocol ${ }^{1}$ was used to document background information, context and nature of the lesson, students, outline of the lesson, classroom culture, use of instructional tools, student tools, facilitation of the tasks, and cognitive demand of the tasks. For the context and nature of the lesson section the observer is required to report the instructional material used and the mathematical strand emphasized during the lesson (which is geometry). The section devoted to students asks for the grade level of students and the total number of students within the classroom. The outline of the lesson requires a description of: the goals, structure and flow, and how reasoning and proof was integrated. For the classroom culture the observer measures the extent the classroom learning environment and mathematical norms provides students an opportunity to learn the mathematical objective of the lesson. The scale used ranged from 1-greatly inhibited to 4-greatly facilitated. Similarly, the use of instructional tools and facilitation of the tasks gauged the extent the tools teachers used facilitate students' opportunity to learn. In the cognitive demand section of the protocol, the observer measured the intellectual potential and engagement of the tasks. A section was added to the protocol to document the facilitation of proof schemes. All of the enacted lessons were audio and video recorded. The teachers carried the audio recorder with them as they move to various groups, to ensure discourse among the students and teachers are recorded, which may not always be captured on the video recording. The video recording focused on board work and teacher actions. Additionally, running field notes were taken of critical timestamps, tasks that might not have been clear on the recording, and other activities that may need to be unpacked further during informal interviews.

1\&2 The observation protocol and artifact packet was developed by Horizon Research, Inc. for the Cases of Reasoning and Proving (CORP) in Secondary Mathematics Project) with funding from the National Science Foundation (Award No. DRL0732798). CORP seeks to develop curriculum that can be used for professional education that promotes reasoning and proving, and the development of mathematical knowledge needed for teaching. Minor adaption was made to the observation protocol, which excluded sections not pertinent to the study, and the section on proof schemes was added. 
To triangulate data recorded on the observation protocol another researcher observed multiple lessons. Our observational results were relatively similar.

\section{Teacher Artifacts}

The teachers were asked to provide copies of handouts distributed to students (i.e. Chapter outlines of pacing guides), samples of students' work that reflected a spectrum of the quality of submission, and to reflect on the planning of the lesson via a task cover sheet-before implementation and task cover sheet-after implementation $^{2}$. For the task cover sheet-before implementation, teachers described the goal and source of the tasks they intend to pose during the enacted lesson, the extent they believed students can engage in constructing proof or a mathematical arguments, propose conjectures or observe noticeable patterns. Whereas, the tasks cover sheet-after implementation, the teachers reflected on the enacted lessons. Teachers discussed how students completed the tasks, alternation made to the tasks, and the nature of communication they had with the students.

\section{Data Analysis}

Prior to observing classroom instruction a textbook analysis was conducted on the visibility of tasks features and cognitive demand of the mathematical tasks within the textbooks for the three chapters examined. Task features considered included the number of proof and proof related tasks the presence of a pictorial image (Picture, image or diagram), multiple choice and fill in the blank tasks. Additionally consideration was given to the level of cognitive demands of the tasks. Multiple researchers assisted with coding tasks for levels of cognitive demand. Sears (2014) reported that fill in the blank proof tasks and pictorial images were rather prevalent in the proof tasks assigned in Prentice Hall Chapter 2-4, and most of the proof requires little cognitive rigor. There existed an $89 \%$ inter-rater reliability among researchers in the classification. This data provided insight into task attributes and the potential cognitive rigor of geometrical tasks teachers would pose.

Subsequently, the pacing guide was analyzed by utilizing descriptive statistics. The frequencies of the number of mathematical tasks and the amount of proof tasks assigned for each section were generated. Additionally, the number of proof tasks assigned was subsequently compared to the total amount of proof tasks within each section.

Data obtained from teacher artifacts, classroom observation protocol, the audio recorded lesson, were imported into NVivo 9 qualitative software and were analyzed using grounded theory approach (Glaser, 2009). Using grounded theory, I sought to generate conclusion by examining each case for inherent features and subsequently engaged in a cross case analysis.

The enacted lessons were coded for tasks features, cognitive demand of enacted tasks, and factors influencing the teaching of proof. For the task features the general codes were: mathematical communication, multiple representations, and multiple solution strategies. The cognitive demands of the tasks were coded as memorization, procedures without connections, procedures with connections, or doing mathematics. For the factors influencing teaching of proof the initial codes considered factors that influence set up and factors that influence students implementation of the task (Henningsen, 1997); however new categories emerged based on the frequency of words and phrases used during the lessons. Hence the codes used for the factors influencing the teaching of proof were: assessment, classroom norm, community (professional environment), making mathematics easy, proof and mathematical tasks should be short, task conditions, students disposition, teachers' beliefs, teachers decision to adapt or improvise the curriculum, teachers' knowledge of students and or students learning, and teachers use of textbook and tools. For this paper, I focus on the data pertinent to the impact the community had on teachers' instructional practices.

\section{RESULTS}

To report the results, I will illustrate the mathematical tasks assigned via the pacing guide in relation to tasks frequencies for the sections in Chapters 2-4 of the textbook (Table 1). Subsequently, I will describe teachers' instructional practices and the extent the pacing guide influenced teachers' practices during the enacted lessons. 
Table 1. The amount of proof tasks assigned in relations to the total amount of tasks by the co-planning initiative, and the total amount of proof tasks within the various sections

\begin{tabular}{cccc}
$\begin{array}{c}\text { Chapters 2-4 } \\
\text { Sections }\end{array}$ & $\begin{array}{c}\text { Number of Proof Tasks } \\
\text { Assigned in the Pacing } \\
\text { Guide }\end{array}$ & $\begin{array}{c}\text { Number of Proof Tasks } \\
\text { within Prentice Hall } \\
\text { Chapters 2-4 Sections }\end{array}$ & $\begin{array}{c}\text { Total Tasks Posed in the } \\
\text { Pacing Guide }\end{array}$ \\
\hline 2.1 & 0 & 0 & 30 \\
\hline 2.2 & 0 & 0 & Not assigned \\
\hline 2.3 & 0 & 0 & 28 \\
\hline 2.4 & 5 & 10 & 27 \\
\hline 2.5 & 1 & 5 & 26 \\
\hline 3.1 & 2 & 5 & 29 \\
\hline 3.2 & 5 & 9 & 31 \\
\hline 3.3 & 2 & 2 & 32 \\
\hline 3.4 & 0 & 0 & 40 \\
\hline 3.5 & 0 & 0 & 33 \\
\hline 3.6 & 0 & 0 & 36 \\
\hline 4.1 & 0 & 0 & 27 \\
\hline 4.2 & 4 & 6 & Not assigned \\
\hline 4.3 & 5 & 6 & 19 \\
\hline 4.4 & 0 & 7 & 16 \\
\hline 4.5 & 2 & 5 & 16 \\
\hline 4.6 & 5 & 10 & 413 \\
\hline 4.7 & 5 & 14 & \\
\hline Total & 36 & 79 & \\
\hline & & & \\
\hline
\end{tabular}

For the pacing guide, across the three chapters, $45.57 \%$ of the 79 proof tasks (Table 1) were assigned to students. Table 1 suggest that students were afforded more opportunities to engage with proof in Chapter 4 , which is devoted to congruent triangles when compared to the other chapters that focused on Reasoning and proof and parallel and perpendicular lines.

\section{Influence of the Pacing Guide on Teachers Planning and Instructional Decisions}

Based on data collected via classroom observations and teacher artifacts, the results indicate that teachers generally adhered to the recommended textbook progression as outlined by the pacing guide because they perceived it was logical and that it ensured students were exposed to content frequently assessed on summative assessment measures. At the beginning of each chapter, both teachers distributed the pacing guide to students, which articulated the tasks they will have to complete for the various sections. Nevertheless, although teachers may assign the same tasks, how they enact lessons may vary. In the subsequent paragraphs, I will discuss how the pacing guide influenced the planning and enactment of proof tasks.

\section{Reduced the cognitive demand of proof tasks}

Based on data collected via the observation protocol, both teachers generally posed proof tasks that require limited cognitive rigor (Sears, 2014), as outlined in the pacing guide. Most of the proof tasks posed were fillin-the-blank tasks, with five or less statements and supporting reasoning. If a more cognitively demanding task was posed, the teachers generally completed the task for the students and required students to take notes about the proof. Their actions generally sought to make the proof easier for students, in an effort to improve the likelihood that students will attempt the proof.

\section{Teachers generally planned to enact textbook tasks as outlined in the pacing guide. If tasks were modified, it was to address the needs of the students}

Both teachers were receptive to following the pacing guide because they believed it worked. They noted that the outline was in place for more than five years, and that teachers can make adjustment to the lessons as needed. 
Mrs. Davis also suggested that her decision to modify a lesson were influenced by her observation of her peers and collegiate experience. For example, Mrs. Davis modified an activity relative to polygons, she stated,

I modified this polygon activity from one I had seen while in college out of a textbook. In the original activity, students are given the sheet with all of the figures and the teacher will read a number and then say yes or no. After several yes and no examples, students are to use reasoning to determine what other figures are yes and no and then work as a group to come up with characteristics of the yes group. The yes group becomes the polygons. (September 23, 2011, task cover-sheet before implementation, Mrs. Davis)

Mrs. Bethel occasionally modified tasks to meet the academic needs of her students. For example, Mrs. Bethel noted,

"This section [Section 2.5- Proving angles congruent] is part of the geometry curriculum and was in place, as agreed upon by the geometry team, before I came to [school name]. Teachers can adjust the assignment as they see fit for their specific needs. The assignment has been the same for my students for the last two terms. I believe it covers the objective well. I originally changed to adjust to the needs of my class and felt the changes would benefit future classes as well." (September 13, 2011, task cover sheet-before implementation, Mrs. Bethel)

Mrs. Bethel changed how the proof was to be written. Instead of having students construct only flow proofs for the lesson, she required students to construct two-column proofs and subsequently use the information in the two-column proofs to construct flow proofs. She noted,

\footnotetext{
"The lesson went as planned. The class is really quite agreeable and seems comfortable with the pace. Students are developing new understanding which is noticeable through discussions at tables, and through notes and homework." (September 13, 2011, task cover sheet-after implementation, Mrs. Bethel).
}

Hence, based on Mrs. Bethel's observation, the modification to review two-column before constructing flow proof appears to be beneficial to students learning. Additionally, Mrs. Bethel was more inclined to pose at least one proof task as a bell work activity (when observed), even if it is not on the outline; this action was not observed in Mrs. Davis class. Nevertheless, students were required to complete the tasks from the textbook that were outline by the pacing guide as homework assignments.

Additionally Mrs. Bethel chose to adjust how she introduced the lesson to ensure the content provided a logical progression to students. For instance, Mrs. Bethel noted, "Geometry team originated the assignment. The modification I made consisted of doing a couple of examples and then having students begin homework [in class]." (September 29 2011, task cover sheet-before implementation, Mrs. Bethel). However, there were instances, in which Mrs. Bethel did not modify the outline. For example, she noted,

\begin{abstract}
Homework assessments were given to me by the geometry team, when I began teaching here. As I have taught this chapter, I have adjusted the assignment a bit, but basically, the assignment is the original. Today's lesson is about side-side-side and side-angle side triangle congruence. NO modifications were made. Specific targets today include SSS and SAS congruence of triangles. Students are probably comfortable with the idea of congruence because we began the chapter discussing congruent figures. Most of the time congruent just makes sense to students, but beginning with this section we start looking closely at the pieces of the triangles. (October 13, 2011, task-cover-sheet-after implementation, Mrs. Bethel).
\end{abstract}

\title{
Departmental norms and state assessment relative to proof
}

The teachers often noted that their plans were to align with the daily objectives of the pacing guide and often noted the department expectation to students. The students were abreast of the expectations considering the pacing guide for tasks to be completed was provided at the beginning of each chapter.

It was customary for teachers to follow the textbook organizational structure and recommendations of means to enact proof tasks. For example, Mrs. Davis noted,

I wouldn't say that I teach the best way, or anything. But ... the way that the book lays out proofs is how we model proofs on our test and on our homework. And the test aren't designed by me, they're designed as a team. (September 23, 2014, Interview, Mrs. Davis) 
Furthermore, the department generally assigned fill-in-the-blank proof tasks. Mrs. Davis, noted,

To do it right, well we set them up to be able to do the proof. Because ... we don't have them construct proofs from A to B on their own typically. It's a fill in the blank; it's a word bank they should be able to. We don't try to trick them. It's not a high level proof that we are teaching. (September 2, 2011, Initial Interview, Mrs. Davis)

Mrs. Bethel echoed Mrs. Davis sentiments. Mrs. Bethel noted,

Students also had to fill-in the blank proofs over review materials. This is what we expect of them. It is important to keep revisiting proofs. Proof is taught differently in different schools. Here at [school name], We teach the basics of proof and do so with the idea that students can master proof given a skeleton and word bank to fill in blanks. I still prefer to work several proofs from scratch and without word banks to try to convince students that the can do it. School Level (geometry team) has dictated that I check for understanding in the classroom. State assessment do not require students to write the proof from scratch, instead, they present the proof as a set of statements and reasons, and have the student "assemble" them in the correct order. (September 22, 2011, task-cover-sheet-after implementation, Mrs. Bethel)

Due to the pacing guide of having students write proof that require them to fill-in-the-blanks, and providing a word bank to help students fill-in-the-blanks potentially reduced the cognitive rigor associated with proof tasks. Furthermore, because the state seldom required students to construct proofs, the value placed on having students construct complete proof may have been compromised. For instance, Mrs. Bethel saw value in having students construct complete proof arguments; yet, she conformed to the departmental norms of having students complete proof by filling in the blanks for homework assignments.

Coding congruent triangles was also a departmental expectation. According to Mrs. Bethel, "When students draw their triangles they must "code and label" them. This is part of the expectations set forth by the geometry team" (October 13, 2011, task cover-sheet after implementation, Mrs. Bethel). She noted that,

\begin{abstract}
Students were able to use proof in this section [4.2- triangle congruence by SSS and SAS). Triangles are proven congruent by SSS and SAS. Reasoning is also practiced through triangles with marks already given for specific sides and/or angles, but student must also use prior knowledge of the reflexive property to recognize segments used in two triangles, as well as vertical angles. These last two ideas are typically not marked in the picture, but we as the geometry team, expect them to code the triangles accordingly. (October 13, 2011, task cover-sheet after implementation, Mrs. Bethel)
\end{abstract}

Hence, the pacing guide readily sought to ensure common expectation across courses and readily aligned with summative assessment measures.

\title{
Teachers' experience provided alternative approaches of how to enact lessons
}

Mrs. Davis and Mrs. Bethel teaching experience influenced the extent they would deviate from the pacing guide. Mrs. Davis acknowledged that this is the only school she taught at and she generally learned to teach proof by observing her peers. If Mrs. Davis modified a task it might have been influenced by observation of her peers and her own teaching experience.

I would say that since this is my six years of teaching. When I first started teaching I wasn't very good at it. But I would say that I have improved. Just having seen other people and observe other teachers and teaching of proofs and their examples of how they teach, I guess I should say. (September 23, 2014, Interview, Mrs. Davis)

Additionally, Mrs. Davis noted,

I found my notes from examples in the book, colleagues, and some were made by me. The homework assignment was problems from the book turned into a worksheet. The original assignment was out of the book, but students did not like to copy the conditional statements out of the book to then underline the hypothesis and conclusion... (September 6, 2011, task cover-sheet before implementation, Mrs. Davis). 
Mrs. Bethel taught at multiple schools and had learned various strategies to enhance her lessons. For example, Mrs. Bethel was inclined to use Kagan resources. She noted,

Activities have been taken from Kagan publishing resources to practice concepts in greater detail. I added the activities from previous geometry teaching experience because I believe the activities help students practice and understand more. Today's lesson focused on the interior and exterior angles of polygons. (September 27, 2011, task cover-sheet after implementation, Mrs. Bethel)

Additionally, Mrs. Davis noted she drew on the activities from a colleague.

\begin{abstract}
I put together a variety of review materials for today. The "complete the chart" activity was suggested by a former teacher. The "boss/ secretary" activity is courtesy of Becky Bride- geometry- Kagan cooperative learning series. The review worksheet is a conglomeration of several concepts that will be tested. (September 15, 2011, task cover-sheet before implementation, Mrs. Bethel)
\end{abstract}

Therefore, the teachers' experiences and personal interactions with students influenced their perspectives about means to modify and enhance lessons outlined in the pacing guide.

\title{
Students' difficulty with proof influenced the enacted lesson
}

Despite the pacing guide, which identified proof tasks that should be assigned, there was no guarantee that students would actually complete the assignments. Both teachers noted that students generally did not complete proof tasks on homework. For example, during the initial interview Mrs. Davis noted, "Well they have a hard time explaining why they did something. A lot of times students don't like to show their work and how did you get to that answer "(September 2, 2011, Initial Interview, Mrs. Davis).

Mrs. Bethel noted that students experience difficulty with corresponding parts of congruent triangles. She stated,

The proof required of the students usually cause confusion. The concept of corresponding parts of congruent triangles are congruent is not difficult by itself really, but it is combined with overlapping triangles which does cause difficulty. CPCTC and overlapping triangles are the main targets of this lesson. The lesson will consist of reasoning and problems and proof, most of which are skeletons that must simply be completed by students. Hopefully, the students are comfortable now with proving triangles congruent, and then adding CPCTC is a simple step. Pulling apart overlapping triangles is difficult for some... (October 25, 2011, task cover-sheet before implementation, Mrs. Bethel).

No matter how I stress this, there will be several who won't code and label or who will not be able to draw the over-lapping triangles. This is a difficult lesson with the geometry students at this school...The goal is to get students to develop the understanding of CPCTC and overlapping triangles...The goal is not always reached... There were definitely proof to complete in this section.... now whether or not the students made these mathematics arguments is a whole different thing! But seriously, yes proof and reasoning are used in this homework assignment. When homework assignment was collected, I was extremely disappointed in the class. More than half the class did not complete the assignment. Unfortunately, I was not surprised. (October 25, 2011, task cover-sheet after implementation, Mrs. Bethel)

Furthermore, Mrs. Bethel noted, "Time does not always work with me" (September 22, 2011, task coversheet before implementation, Mrs. Bethel).

Evidently, teachers were aware that students were not inclined to complete proof tasks on homework, and that students may experience difficulty with proving. However, due to time constraints, teachers were challenged to meet other objectives rather than increase greater time allocation for proof, and accepted the notion that students generally avoid proving for homework assignments.

Additionally, Mrs. Davis noted that students did not enjoy writing long proof and often chose to abbreviate mathematical theorems and postulates. Mrs. Davis noted, "I had a lot of students in previous years, not do the assignment because there was too much writing" (September 6, 2011, task cover-sheet before implementation, Mrs. Davis). Hence, she allowed students to write in an abbreviated form. For example,

I told students that they could abbreviate their classifications for polygons even though that was not included on the task itself. EL for equilateral, EA for equiangular, CC for concave, etc. (September 23, 2011, task cover-sheet after implementation, Mrs. Davis). Additionally, based on classroom observation, students 
were allowed to refer to theorem as Theorem 4-1, 4-2, rather than explicitly state the theorem, which can be difficult to interpret if a copy of the textbook is not available.

\section{DISCUSSIONS}

The pacing guide promoted consistency in the coverage of mathematical ideas within an academic semester, across mathematical classrooms. Based on the pacing guide, slightly less than half of the proof tasks within the three chapters were assigned and little time was allocated to unpack proof tasks, considering there were many objectives that had to be met. Teachers sought to address the identified objectives, within the specified timeframe, by posing similar (but not necessarily the same) tasks. The common homework assignments allowed students to practice and review core concepts, and provided teachers a means to evaluate the extent their students are comparable to their counterparts in other class for various topics in the textbook. The pacing guide can potentially have merits for summative assessment purposes, however, it cannot guarantee the depth of mathematical understanding facilitated within each classroom considering the differences in the enacted lessons, classroom culture and the various learners within the classroom environment.

Despite teachers' adherence to the pacing guide, in which they assigned the homework and practice tasks, the teachers varied in tasks they explicitly used during the enacted lessons, and the attention to precision during the classroom discourse. Salient factors, such as the depth of teachers' knowledge and experience with teaching proof contributed to the decisions teachers ultimately made relative to the enacted lessons. For example, Mrs. Bethel had more teaching experience than Mrs. Davis, and was mindful of potential differences in the language and tasks within textbooks. She supplemented tasks from Kaagan mathematics and added proofs to her lessons when observed. The decision to supplement the textbook with alternative tasks provided increased opportunity for students to work in pairs since the activities explicitly required students to do so. Furthermore, the inclusion of proof tasks to lessons increased instances in which students engaged in proof. Admittedly, the rigor of the extra proof may be limited, however there is still value in students observing a proof in lessons that were not devoted to proving. Researchers can potentially examine the implication of teachers posing a proof task each day because it can circumvent the perception that proof is topic specific, rather than a mathematical process that should be encouraged across the curriculum.

Furthermore, Mrs. Bethel required students to carefully articulate theorems, whereas Mrs. Davis allowed students to abbreviate theorems, which at times was not understood by an individual outside of the community. Considering the subjectivity of what constitutes a proof, and that little time is allocated to prove, it is important that attention to precision is given to the communication of mathematical constructs when students are afforded the opportunity to prove. The language used within the classroom setting should be understood and interpreted across multiple settings. Thus, although abbreviation and textbook reference to theorems (such a theorem 4.1,4.2, etc) can reduce the time needed to write a proof, such practices can potentially hinder students' development of mathematical articulation about reasoning to support the proof. Textbooks can change; hence students must be able to explicitly state the theorem such that a wider community could understand their ideas. Therefore, in the construction of arguments, greater attention ought to be placed on the articulation of axioms, theorems, definitions and postulates. The nature of discourse should seek to promote verbal fluency in mathematical ideas as well as strengthen the quality of the depth of reasoning used to support claims made within the proof.

The pacing guide amplified the interplay between the textbook and the professional environment. Teachers noted that limited attention was allocated to proof within the textbook and that most of the proofs assigned required minimum cognitive rigor. Nevertheless, the teachers were receptive to the inherent features of the textbook, and acknowledged that the sequential order of topics within the book logically linked mathematical ideas together. Additionally, the teachers were aware of the accountability implications at the school for students' performance on end of semester assessment performance in geometry. Thus, teachers sought to ensure that the pedagogical emphasis in the textbook positively impacted students learning (which is evaluated), and complemented their professional agency.

The teachers had the capacity to make changes to the tasks assigned in the pacing guide, to meet the needs of their particular students. This flexibility provided the opportunity for teachers' agency in the enacted lessons to manifest. Davies (1991) noted, "Agency is never freedom from discursive constitution of self but the capacity to recognise that constitution and to resist, subvert and change the discourses themselves through which one is being constituted". Teachers could have chosen to deviate from the textbook and increase 
opportunities for students to prove, however, the teachers chose to adhere to the textbook content progression. The nature of the classroom discourse focused on the chapters' big idea and review of content were that were readily assessed. Perhaps, if proof was assessed more readily the likelihood for teachers to place a greater emphasis on students' construction of proof may increase. Although teachers acknowledge the benefits of proof the value placed on proof in the classroom setting was minimal.

Moreover, the teachers bartered the cognitive demand of proof tasks assigned for students to complete the proof. Generally, the teachers posed lower levels of cognitive demand and if the task had the potential to require some degree of rigor the teachers completed the proof. This decision might have been influenced by the teachers' pedagogical content knowledge of students and their disposition towards proving. The strategy of direct instruction provides students with explicit information needed to construct the proof. Being mindful that the students were not inclined to prove, by telling the students the information was disseminated and students had less reasons to complain about the amount of thinking required to construct proof. This practice can transcend multiple topics and is not restricted to proof.

\section{CONCLUSION}

The teachers adhered to the instructional recommendations suggested by their geometry team. If a teacher utilized alternative materials, the tasks were rather similar to the suggested textbook tasks. Proof tasks recommended and subsequently enacted generally required students to fill in the blanks and were similar to the example in the exposition section of the textbooks. The geometry team made adjustments to proof tasks that required students to construct complete proof by providing a partial structure. Thus, the rigor required to complete potentially rich tasks was often reduced.

Notwithstanding, that there are benefits in planning as a group, consideration ought to be given as to what tasks are emphasized and the amount of time allocated for particular topics. Not all tasks in the textbooks are assigned, thus, the need to unpack the decision as to what is assigned and how such tasks are eventually enacted in the classroom can have implication on the mathematics students ultimately learns. In the context of geometry, students were seldom assigned proof and if it were assigned the teachers primarily did the proof for the students. As a result, students were provided little opportunity to engage in the mathematical process of proving.

The tasks recommended and subsequently implemented minimized opportunities for students to construct proof in its entirety, or engage in rigorous proving activities. Thus, the pacing guide can potentially influence how proof is taught and learned in a negative way, if only few tasks requires cognitive rigor. Therefore, geometry teams need to increase the amount of proof tasks to which students are exposed and the opportunity for students to write complete proofs.

\section{Disclosure statement}

No potential conflict of interest was reported by the authors.

\section{Notes on contributors}

Ruthmae Sears - University of South Florida.

\section{REFERENCES}

Alibert, D., \& Thomas, M. (1991). Research on mathematical proof Advanced mathematical thinking (pp. 215230): Springer.

Bass, L. E., Charles, R. I., Johnson, A., \& Kennedy, D. (2004). Prentice hall mathematics geometry. Upper Saddle River, NJ: Pearson Education, Inc.

Blanton, M. L., \& Stylianou, D. A. (2009). Interpreting a community of practice perspective in disciplinespecific professional development in higher education. Innovative Higher Education, 34(2), 79-92. https://doi.org/10.1007/s10755-008-9094-8

Brown, J., \& Stillman, G. (2009). Preservice secondary teachers' competencies in proof. in Mathematics Education, 94. 
Brown, T., \& McNamara, O. (2011). Theorising teacher identity becoming a mathematics teacher (pp. 87-109): Springer. https://doi.org/10.1007/978-94-007-0554-8

Cobb, P., McClain, K., de Silva Lamberg, T., \& Dean, C. (2003). Situating teachers' instructional practices in the institutional setting of the school and district. Educational Researcher, 32(6), 13-24. https://doi.org/10.3102/0013189X032006013

Davies, B. (1991). The concept of agency. Social Analysis, 30, 42-53

Donoghue, E. F. (2003). Algebra and geometry textbooks in twentieth-century America. A history of school mathematics, 329-398.

Franke, M. L., \& Kazemi, E. (2001). Teaching as learning within a community of practice: Characterizing generative growth. Beyond classic pedagogy: Teaching elementary school mathematics, 47-74.

Furinghetti, F., \& Morselli, F. (2011). Beliefs and beyond: Hows and whys in the teaching of proof. ZDM, 43(4), 587-599. https://doi.org/10.1007/s11858-011-0316-7

Fussell, K. (2005). The educational purposes of geometric proof in the high school curriculum (Master's Degree Thesis), North Carolina State University, Raleigh, North Carolina.

Glaser, B. G., \& Strauss, A. L. (2009). The discovery of grounded theory: Strategies for qualitative research: Transaction Books.

Grouws, D. A., \& Smith, M. S. (2000). NAEP findings on the preparation and practices of mathematics teachers. Results from the seventh mathematics assessment of the National Assessment of Educational Progress, 107-139.

Grouws, D. A., Smith, M. S., \& Sztajn, P. (2004). The preparation and teaching practices of United States mathematics teachers: Grades 4 and 8 (pp. 221-269). Reston, VA: National Council of Teachers of Mathematics.

Healy, L., \& Hoyles, C. (2000). A study of proof conceptions in algebra. Journal for Research in Mathematics Education, 31(4), 396-428. https://doi.org/10.2307/749651

Heinze, A., \& Reiss, K. (2009). Developing argumentation and proof competencies in mathematics classroom In D. A. Stylianou, M. L. Blanton \& E. J. Knuth (Eds.), Teaching and learning proof across the grades. New York Routledge.

Henningsen, M., \& Stein, M. K. (1997). Mathematical tasks and student cognition: Classroom-based factors that support and inhibit high-level mathematical thinking and reasoning. Journal for Research in Mathematics Education, 28(5), 524-549. https://doi.org/10.2307/749690

Herbst, P. G. (2002). Establishing a custom of proving in American school geometry: Evolution of the twocolumn proof in the early twentieth century. Educational Studies in Mathematics, 49(3), 283-312. https://doi.org/10.1023/A:1020264906740

Herbst, P. G., Chen, C., Weiss, M., Gonzalez, G., Nachlieli, T., Hamlin, M., \& Brach, C. (2009). "Doing proofs" in geometry classrooms. In D. A. Stylianou, M. L. Blanton \& E. J. Knuth (Eds.), Teaching and learning proof across the grades (pp. 250-268). New York Routledge.

Jones, K. (2000). Critical issues in the design of the school geometry curriculum. In B. Barton (Ed.), Readings in mathematics education (pp. 75-90). Auckland, New Zealand: University of Auckland.

Jones, K. (2002). Issues in the teaching and learning of geometry. In L. Haggarty (Ed.), Aspects of teaching secondary mathematics: Perspectives on practice. (pp. 121-139). London, UK: Routledge Falmer. https://doi.org/10.1002/tl.62

Knuth, E. J. (2002). Secondary school mathematics teachers' conceptions of proof. Journal for Research in Mathematics Education, 33(5), 379-405. https://doi.org/10.2307/4149959

Knuth, E. J., Choppin, J., \& Bieda, K. (2009). Middle school students' production of mathematical justifications. In D. A. Stylianou, M. L. Blanton \& E. J. Knuth (Eds.), Teaching and learning proof across the grades. New York: Routledge.

Lloyd, G., Remillard, J. T., \& Herbel-Eisenmann, B. (2009). Teachers' use of curriculum materials: An emerging field. In J. T. Remillard, B. A. Herbel-Eisenmann \& G. M. Lloyd (Eds.), Mathematics teachers at work (pp. 3-14). New York: Routledge.

Martin, T. S., McCrone, S. M. S., Bower, M. L. W., \& Dindyal, J. (2005). The interplay of teacher and student actions in the teaching and learning of geometric proof. Educational Studies in Mathematics, 60(1), 95124. https://doi.org/10.1007/s10649-005-6698-0 
Philipp, R. A. (2007). Mathematics teachers. Beliefs and A ect,Äô. In: FK Lester, Jr.(ed.): Second Handbook of Research on Mathematics Teaching and Learning. Charlotte, NC: Information Age Publishing, 257-315.

Remillard, J. T. (2005). Examining key concepts in research on teachers' use of mathematics curricula. Review of Educational Research, 75(2), 211-211. https://doi.org/10.3102/00346543075002211

Remillard, J. T. (2009). Part ii commentary: Considering what we know about the relationship between teachers and curriculum materials (pp. 85-92). New York: Routledge.

Schwarz, B. R., \& Kaiser, G. (2009). Professional competence of future mathematics teachers on argumentation and proof and how to evaluate it. Mathematics Education, 190.

Sears, R., \& Chávez, Ó. (2014). Opportunities to engage with proof: The nature of proof tasks in two geometry textbooks and its influence on enacted lessons. ZDM, 46(5), 767-780.

Spillane, J. P. (2000). A fifth-grade teacher's reconstruction of mathematics and literacy teaching: Exploring interactions among identity, learning, and subject matter. The Elementary School Journal, 307-330. https://doi.org/10.1086/499644

Stein, M. K., Remillard, J. T., \& Smith, M. S. (2007). How curriculum influences student learning. In F. K. J. Lester (Ed.), Second handbook of research on mathematics teaching and learning (Vol. 1, pp. 319-369). Charlotte, NC: Information Age Publishing

Stylianides, G. J. (2008). Investigating the guidance offered to teachers in curriculum materials: The case of proof in mathematics. International Journal of Science and Mathematics Education, 6(1), 191-215. https://doi.org/10.1007/s10763-007-9074-y

Yackel, E., \& Cobb, P. (1996). Sociomathematical norms, argumentation, and autonomy in mathematics. Journal for Research in Mathematics Education, 27(4), 458-477. https://doi.org/10.2307/749877 